\title{
Simultaneous Determination of Ephedrine and Pseudoephedrine in Mice Plasma by Capillary Zone Electrophoresis
}

\author{
Chunmei Zhang \\ College of Pharmacy, Hebei University \\ Baoding 071002, Hebei, China \\ E-mail: zhangcm19850304@126.com \\ Huaizhong Guo (Corresponding author) \\ College of Pharmacy, Hebei University \\ Baoding 071002, Hebei, China \\ E-mail: ghuaizh@yahoo.com.cn \\ Li Guan, Weiquan Zhang, Qian Wen \& Chunying Chen \\ College of Pharmacy, Hebei University \\ Baoding, Hebei, 071002, China
}

The authors gratefully acknowledge the financial support from the National Natural Science Foundation of China (No. 20905019; 21011140338/B0511), Natural Science Foundation of Hebei Province (No. B2010000209) and Natural Science Foundation of Hebei University (No. 2007-111).

\begin{abstract}
A low cost, accurate, rapid and sensitive capillary zone electrophoresis (CZE) quantitative method, using thiamine hydrochloride as internal standard (IS), was developed for the simultaneous analysis of ephedrine and pseudoephedrine in mice plasma. Analytes were extracted from plasma samples by n-hexane/dichloromethane/isopropanol (2:1:0.1, v/v/v) after alkalization, and stripped with 1.5\% hydrochloric acid solution. Separation was performed by CZE using $50 \mathrm{mM}$ borax-20mM threonine ( $\mathrm{pH} 9.27)$ as running buffer, with voltage of $+10 \mathrm{kV}$ applied and UV detection wavelength at $210 \mathrm{~nm}$. The total running time was within 10 min. Calibration curves for plasma samples were linear over the concentration range of $0.075-5.0 \mu \mathrm{g} / \mathrm{mL}$ for ephedrine and $0.0375-2.5 \mu \mathrm{g} / \mathrm{mL}$ for pseudoephedrine. The method had a limit of quantitation of $17.80 \mathrm{ng} / \mathrm{mL}$ for ephedrine and $22.00 \mathrm{ng} / \mathrm{mL}$ for pseudoephedrine,respectively. The method was reliable and feasible for the evaluation of pharmacokinetic profiles of drug products containing ephedrine and pseudoephedrine.
\end{abstract}

Keywords: Ephedrine, Pseudoephedrine, Capillary Zone Electrophoresis

\section{Introduction}

Ephedrine (E) and Pseudoephedrine (PE) are pairs of diastereoisomeric sympathomimetic amines that have human central nervous system stimulating properties, and they also can relieve the spasm of the bronchial smooth muscle (Lou Hong-gang, 2010, PP.682-688, Kang Yong, 2001, PP.37-39). Several methods have been employed for the detection of the individual component in plasma, including HPLC (Aymard G. 2000, P.25, Roman MC. 2004, PP.15-24), LC-MS/MS (Beyer J. 2007, PP.150-160, Yi Feng, 2008, P.572), GC-MS (Gunn J. 2010, PP.37-43, Nakano M. 2000, PP.583-586) and CE (Wei F. 2007, PP.38-44, Liu Gang, 2006, P.617). Recently, a GC-MS/SIM method was reported for the simultaneous determination of $\mathrm{E}$ and $\mathrm{PE}$ in humn plasma through liquid-liquid extraction and derivatization by trifluoroacetic anhydride (TFA) with a lower limit of quantitation (LOQ) of 2 and 1ng/mL for E and PE, respectively (Shen Qun, 2002). Although the method achieved sufficient detection sensitivity in short run time, it suffered from high cost and time-consuming extraction procedure. Therefore, a highly sensitive and simple CZE method was developed and validated for the simultaneous determination of $\mathrm{E}$ and $\mathrm{PE}$ in mice plasma. This developed method offered smaller sample volume requirement and shorter run time, especially lower cost. This method can be flexibly applied to a bioequivalence study. 


\section{Experimental}

\subsection{Materials and reagents}

E was purchased from national institute for the control of pharmaceutical and biological products (Beijing, China), and PE was obtained from national narcotics laboratories (Beijing, China). Thiamine hydrochloride manufactured by the Medical Chemical Reagent (Tianjin, China) was used as the internal standard (IS). HPLC grade methanol was purchased from Kermel Chemical (Tianjin, China). Borax and all the other reagents used in the experiment were all of analytical grade and obtained from Huaxin Chemical Reagent (Baoding, China). Blank mice plasma was obtained from the experimental animals center of Hebei Medical University (Shijiazhuang, China). Ultrapure water was obtained from AK water treatment system (Taiwan, China).

\subsection{Instrumentation}

High Performance Capillary Electrophoresis system (Cailu Co. Ltd, Beijing, China) is with a 0-30 kV high voltage power supply, and a variable wavelength UV detector near the column end. Data acquisition and procession were performed using HW-2000 software (Qianpu Co. Ltd, Shanghai, China). Capillaries with $50 \mu \mathrm{m} \mathrm{I.D.} \mathrm{and} 375 \mu \mathrm{m}$ O.D.(Yongnian Ruifeng Photoconductive Fiber Factory, Hebei, China). Buffer pH was measured with a PHS-3C pH meter (Shanghai, China). A vortex mixer (QL-901, Haimen Medical Instrument, Jiangsu, China) was used to extract the analytes and a centrifuge (L500, Xiangyi Centrifuge Instrument Co. Ltd, Changsha, China) was used to accelerate the separation of organic and inorganic phases.

\subsection{CZE conditions}

Before the experiment, the capillary column should be pretreated. Firstly, the capillary column was rinsed with 1.0 $\mathrm{M} \mathrm{NaOH}$ solution for $30 \mathrm{~min}$ and then with water until the $\mathrm{pH}$ value of the outlet solution reached 7.0. Secondly, the capillary was rinsed with $1.0 \mathrm{M} \mathrm{HCl}$ solution for $30 \mathrm{~min}$ and then with water until the $\mathrm{pH}$ value of the outlet solution reached 7.0.

Then, the conditions of the experiment were optimized with a fused-silica capillary of $50 \mathrm{~cm} \times 40 \mu \mathrm{m}(40 \mathrm{~cm}$ effective length) in a running buffer of $50 \mathrm{mM}$ borax- $20 \mathrm{mM}$ threonine (the $\mathrm{pH}$ value was adjusted to 9.27 with $1 \mathrm{M}$ $\mathrm{NaOH}$ solution) and an applied voltage of $+10 \mathrm{kV}$ at room temperature. Samples were introduced by hydrodynamic injection $(15 \mathrm{~cm} \times 30 \mathrm{~s})$ and the detection wavelength was $210 \mathrm{~nm}$.

\subsection{Preparation of mixed stock and working solutions}

The mixed stock solutions of $\mathrm{E}$ and $\mathrm{PE}$ were prepared together by dissolving accurately weighed reference standards in methanol to result in a final concentration of $1 \mathrm{mg} / \mathrm{mL}$ for $\mathrm{E}$ and $0.5 \mathrm{mg} / \mathrm{mL}$ for PE. Standard working solutions were prepared by diluting the mixed stock solutions with methanol to get six different concentrations in a range of $0.3-20 \mu \mathrm{g} / \mathrm{mL}$ for $\mathrm{E}$ and $0.15-10 \mu \mathrm{g} / \mathrm{mL}$ for PE. IS working solution was prepared with $1.5 \%$ hydrochloric acid solution to the nominal concentration of $10 \mu \mathrm{g} / \mathrm{mL}$.

\subsection{Preparation of standard and quality control samples}

$100 \mu \mathrm{L}$ of each of the above different concentration working solutions was added to $400 \mu \mathrm{L}$ blank mice plasma to obtain the desired series plasma concentration of analytes. The plasma concentrations of calibration standards were $0.075,0.125,0.25,0.5,1.25,5.0 \mu \mathrm{g} / \mathrm{mL}$ for $\mathrm{E}$ and $0.0375,0.0625,0.125,0.25,0.625,2.5 \mu \mathrm{g} / \mathrm{mL}$ for PE, respectively. The quality control (QC) samples were prepared in the same way as the calibration standards to obtain plasma concentrations of $0.125,0.5,5.0 \mu \mathrm{g} / \mathrm{mL}$ for E and $0.0625,0.25,2.5 \mu \mathrm{g} / \mathrm{mL}$ for PE. All of these plasma samples were kept at $-20{ }^{\circ} \mathrm{C}$ before use.

\subsection{Sample preparation}

(1) Liberation and organic solvent extraction

In $15 \mathrm{~mL}$ centrifuge tubes, a standard sample (mentioned aboved) was mixed with $0.5 \mathrm{~mL}, 2 \mathrm{M} \mathrm{NaOH}$, and the analytes were liberated from plasma by vortex-mixing for $1 \mathrm{~min} .4 \mathrm{~mL}$ mixed organic solutions of n-hexane/dichloromethane/isopropanol $(2: 1: 0.1, \mathrm{v} / \mathrm{v} / \mathrm{v})$ was added and vortex-mixed for $3 \mathrm{~min}$, then the analytes diverted into the organic phase. After centrifugation with $5000 \mathrm{rmp}$ for $6 \mathrm{~min}$, the organic phase was transferred to another centrifuge tube.

\section{(2) Back extraction}

$100 \mu \mathrm{L}$ IS working solution was added and the mixture was back extracted by vortex-mixing for $3 \mathrm{~min}$. Discarding the upper organic phase after centrifugation with $5000 \mathrm{rmp}$ for $6 \mathrm{~min}$. The lower aqueous phase was dried in $75^{\circ} \mathrm{C}$ water bath, and then the residues was dissolute in $10 \mu \mathrm{L}$ ultrapure water to obtain the enriched analytes, which was analyzed by CZE subsequently. 


\section{Results and discussion}

\subsection{Optimization of CZE conditions}

The influence of the separation voltage on the separation efficiency was evaluated. The separation voltage at +20 , +15 and $+10 \mathrm{kV}$ were tested. The electropherograms in fig. 1 obviously showed that optimum separation voltage was $+10 \mathrm{kV}$.

With the increasing of the injection time, the intensity of the signals of analytes increased, generally to obtain the high sensitivity. A standard mixture was injected into the capillary at different time between $10 \mathrm{~s}$ and $40 \mathrm{~s}$ by hydrodynamic injection, keeping the sampling height constant at $15 \mathrm{~cm}$. The results indicate that peak areas are proportional to the injection time up to $40 \mathrm{~s}$. However, when an injection time was longer than $30 \mathrm{~s}$, so did peak broadening and worse resolution presented. Thus, an injection time of $30 \mathrm{~s}$ was selected.

\subsection{Selection of sample preparation conditions}

Due to the low concentration of $\mathrm{E}$ and PE in human plasma, the high extraction efficiency and sample enrichment is in need. The developed extraction method could avoid the interference of endogenous compounds and overcome the shortcoming of low recovery rate through the process of basification, extraction followed by acidification. Additionally, the investigation for the organic extractant indicated that the extraction efficiency with the mixture of $n$-hexane-dichloromethane-isopropanol as extractant was obviously higher than that with the cyclohexane-dichloromethane as extractant. Simultaneously, this work studied the effects of the different hydrochloric concentrations $(0.3 \%, 0.5 \%, 1.0 \%, 1.5 \%, 2.0 \%)$ on the extraction efficiency for the analytes. The results indicated that the higher recoveries with the increase of the hydrochloric concentrations, but when $2.0 \%$ hydrochloric solution was used, extraction efficiency has no obvious change compared with $1.5 \%$ hydrochloric solution, so $1.5 \%$ was chosen.

\subsection{Method validation}

\subsubsection{Separation and specificity}

The CZE-based method in this paper was investigated by analyzing the blank plasma sample, spiked plasma samples and mice plasma sample, and their electropherograms were shown in Fig. 2, Fig. 3 and Fig. 4. Under the CZE conditions described above, the migration time was about $7.210 \mathrm{~min}$ for IS, $7.857 \mathrm{~min}$ for E and $9.292 \mathrm{~min}$ for $\mathrm{PE}$, respectively. There were no interference peaks found at the migration times of the two analytes and IS.

\subsubsection{Linearity}

Calibration curves for plasma were good linear over the concentration range of $0.075-5.0 \mu \mathrm{g} / \mathrm{mL}$ for $\mathrm{E}$ and $0.0375-2.5 \mu \mathrm{g} / \mathrm{mL}$ for PE. Typical equations of calibration curves were as follows: for $\mathrm{E}, \mathrm{C}=2.688 \mathrm{~A}+0.1999$ $(\mathrm{r}=0.9982)$, and for $\mathrm{PE}, \mathrm{C}=3.322 \mathrm{~A}+0.1418(\mathrm{r}=0.9988)$, where $\mathrm{A}=$ peak-area ratio and $\mathrm{C}=$ concentration $(\mu \mathrm{g} / \mathrm{mL})$.

\subsubsection{LOQ and LOD}

The current assay had a LOQ calculated on the basis of a signal-to-noise $(\mathrm{S} / \mathrm{N})$ ratio of $10: 1$ were $17.18 \mathrm{ng} / \mathrm{mL}$ for E, and $22.00 \mathrm{ng} / \mathrm{mL}$ for PE. The LODs were $5.34 \mathrm{ng} / \mathrm{mL}$ for E and $6.60 \mathrm{ng} / \mathrm{mL}$ for PE $(\mathrm{S} / \mathrm{N}=3)$. These limits are sufficient for clinical pharmacokinetic studies after oral administration of therapeutic dose.

\subsubsection{Intra-day and inter-day precisions}

The precision of the method was evaluated by analyzing five replicates of QC samples at low, medium and high concentrations, respectively. Intra-day precision was assessed as the relative standard deviation (RSD) resulting from the same day. Inter-day assay precision was expressed by the RSD of the mean concentrations on three consecutive days. The summary of intra-day and inter-day precisions at QC concentrations was shown in Table 1. The results were less than $5.30 \%$ and $6.75 \%$ for the two analytes, which indicated good precision.

\subsubsection{Recovery}

The recovery experiment was carried out to evaluate the accuracy of the method. In this study, the recovery was determined from QC samples of low, medium and high concentrations, and recovery $\%=$ measured concentration/ $\mathrm{QC}$ sample concentration $\times 100 \%$. Table 2 summarized the results of recoveries of the two analytes, which were in range of $97.6-106.9 \%$ and RSD were in range of $2.46-8.29 \%$. Thus, recoveries were within the acceptable criteria.

\section{Conclusions}

In our study, a low cost and accurate CZE method with high sensitivity was developed . The satisfactory results of the methodology validation indicated that the method can be applied to simultaneous determination of $\mathrm{E}$ and PE plasma concentrations in complex clinical samples, for example, human plasma and herbal medicine. 


\section{References}

Aymard G, Labarthe B \& Warot D. (2000). Sensitive determination of ephedrine and norephedrine in human plasma samples using derivatization with 9-fluorenylmethyl chloroformate and liquid chromatography. Chromatogr, B, 01, 25.

Beyer, J., Peters, FT., Kraemer, T. \& Maurer, HH. (2007). Detection and validated quantification of nine herbal phenalkylamines and methcathinone in human blood plasma by LC-MS/MS with electrospray ionization. Journal of Mass Spectrometry, 02, 150-160.

Gunn J, Kriger S \& Terrell AR. (2010). Identification and quantitation of amphetamine, methamphetamine, MDMA, pseudoephedrine and ephedrine in blood, plasma and serum using gas chromatography-mass spectrometry (GC/MS). Methods Mol Biol., 607, 37-43.

Kang Yong. (2001). Chinese Pharmacology. Beijing: Science Press, PP. 37-39.

Liu Gang, Zhang Hui \& Zhang Xianzhou. (2006). Determination of pseudoephedrine in human serum by HPCE. China Pharmacist, 07, 617.

Lou Hong-gang, Yuan Hong, Ruan Zou-rong \& Jiang, Bo. (2010). Simultaneous determination of paracetamol, pseudoephedrine, dextrophan and chlorpheniramine in human plasma by liquid chromatography-tandem mass spectrometry. Chromatography B., 878, 682-688.

Nakano M, Morimoto Y, Tajima S \& Kosaka N. (2000). GC-MS determination of 1-ephedrine and d-pseudoephedrine in human plasma. Yakugaku Zasshi, 06, 583-586.

Roman MC. (2004). Determination of ephedra alkaloids in urine and plasma by HPLC-UV: Collaborative study. Journal of AOAC International, 01, 15-24.

Shen Qun. (2002). the effect of the different compatibility of mahuangtang on ephedrine and pseudoephedrine pharmacokinetics in mice. First Military Medical University.

Wei F, Zhang M \& Feng YQ. (2007). Combining poly (methacrylic acid-co-ethylene glycol dimethacrylate) monolith microextraction and on-line pre-concentration-capillary electrophoresis for analysis of ephedrine and pseudoephedrine in human plasma and urine. J Chromatogr B Analyt Technol Biomed Life Sci, 01-02, 38-44.

Yi Feng, Yu Huang \& Lai Weiling. (2008). Determination of pseudoephedrine in rabbit plasma by LC-MS/MS. Journal of Guangdong College of Pharmacy, 06, 572.

Table 1. Intra-day and inter-day precision for E from plasma samples $(\mathrm{n}=5)$

\begin{tabular}{|c|c|c|c|c|c|}
\hline \multirow{2}{*}{\multicolumn{2}{|c|}{$\begin{array}{c}\text { Concentratio } \\
\text { added } \\
(\mu \mathrm{g} / \mathrm{mL})\end{array}$}} & \multicolumn{2}{|l|}{ Intra-day $(n=5)$} & \multicolumn{2}{|c|}{ Intra-day $(n=5)$} \\
\hline & & $\begin{array}{c}\text { Measured concentration } \\
(\mu \mathrm{g} / \mathrm{mL})\end{array}$ & $\begin{array}{l}\text { Precision } \\
(\text { RSD \%) }\end{array}$ & $\begin{array}{c}\text { Measured concentration } \\
(\mu \mathrm{g} / \mathrm{mL})\end{array}$ & $\begin{array}{l}\text { Precision } \\
\text { (RSD \%) }\end{array}$ \\
\hline \multirow[b]{3}{*}{$\mathrm{E}$} & 0.125 & $0.139 \pm 0.003$ & 2.33 & $0.120 \pm 0.007$ & 6.04 \\
\hline & 0.5 & $0.447 \pm 0.016$ & 3.50 & $0.478 \pm 0.021$ & 4.30 \\
\hline & 5.0 & $4.966 \pm 0.073$ & 1.46 & $5.021 \pm 0.134$ & 2.66 \\
\hline \multirow[b]{3}{*}{$\mathrm{PE}$} & 0.0625 & $0.067 \pm 0.004$ & 5.30 & $0.062 \pm 0.004$ & 6.75 \\
\hline & 0.25 & $0.232 \pm 0.010$ & 4.48 & $0.261 \pm 0.011$ & 4.40 \\
\hline & 2.5 & $2.516 \pm 0.064$ & 2.53 & $2.413 \pm 0.110$ & 4.55 \\
\hline
\end{tabular}

Table 2. Recoveries for $\mathrm{E}$ and $\mathrm{PE}$ for $\mathrm{E}$ from plasma samples $(\mathrm{n}=5)$

\begin{tabular}{|c|c|c|c|c|}
\hline \multicolumn{2}{|c|}{$\begin{array}{c}\text { Concentration } \\
\text { Added }(\mu \mathrm{g} / \mathrm{mL})\end{array}$} & $\begin{array}{c}\text { Measured } \\
\text { Concentration }(\mu \mathrm{g} / \mathrm{mL})\end{array}$ & $\begin{array}{c}\text { Recovery } \\
\pm \mathrm{S}(\%)\end{array}$ & $\begin{array}{c}\text { RSD } \\
(\%)\end{array}$ \\
\hline \multirow{4}{*}{$\mathrm{E}$} & 0.125 & $0.134 \pm 0.009$ & $106.9 \pm 6.94$ & 6.50 \\
\cline { 2 - 5 } & 0.5 & $0.489 \pm 0.018$ & $97.8 \pm 3.69$ & 3.78 \\
\cline { 2 - 5 } & 5.0 & $5.063 \pm 0.183$ & $101.3 \pm 3.66$ & 3.61 \\
\hline \multirow{4}{*}{$\mathrm{PE}$} & 0.0625 & $0.061 \pm 0.005$ & $97.6 \pm 8.08$ & 8.29 \\
\cline { 2 - 5 } & 0.25 & $0.246 \pm 0.006$ & $98.3 \pm 2.42$ & 2.46 \\
\cline { 2 - 5 } & 2.5 & $2.520 \pm 0.131$ & $100.8 \pm 5.24$ & 5.20 \\
\hline
\end{tabular}




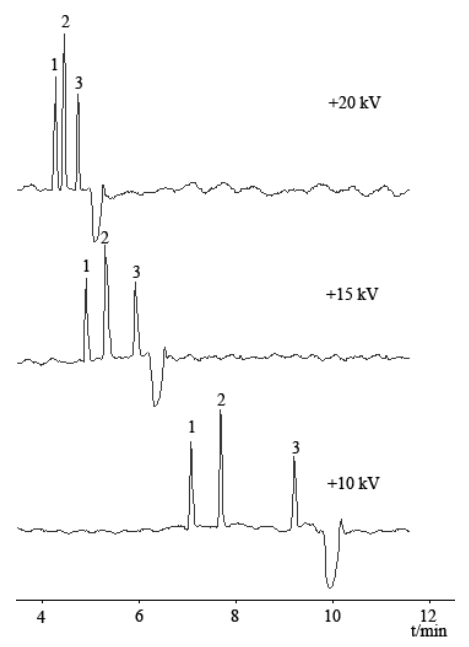

Figure 1. The eletropherograms of analytes were at different separation voltage Peak identification: (1) IS; (2) E; (3) PE

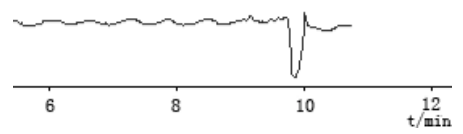

Figure 2. The eletropherogram of a blank plasma

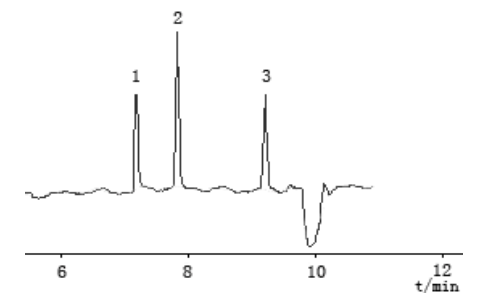

Figure 3. The eletropherogram of a blank plasma sample was added with E, PE and IS Peak identification as in Fig. 1

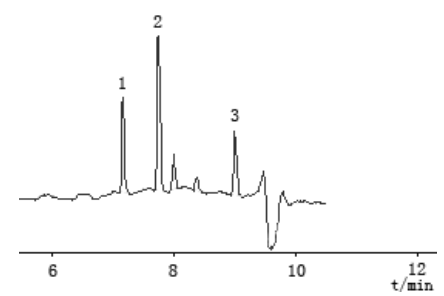

Figure 4. The electropherograms of a plasma sample from the mice after $2 \mathrm{~h}$ of $i g$ Maxingshigan Decoction. Peak identification as in Fig. 1 